\title{
ON A DOUBLE BIFURCATION QUASILINEAR PROBLEM ARISING IN THE STUDY OF ANISOTROPIC CONTINUOUS MEDIA
}

\author{
FLORICA-CORINA ŞT. CÎRSTEA AND VICENŢIU D. RĂDULESCU \\ Department of Mathematics, University of Craiova, \\ 1100 Craiova, Romania (radules@ann.jussieu.fr)
}

(Received 16 March 2000)

Abstract We study the bifurcation problem

$$
\begin{cases}-\operatorname{div}\left(a(x)|D u|^{p-2} D u\right)+h(x) u^{r-1}=f(\lambda, x, u) & \text { in } \Omega \subset \mathbb{R}^{N}, \\ a(x)|D u|^{p-2} D u \cdot n+b(x) u^{p-1}=\theta g(x, u) & \text { on } \Gamma, \\ u \geqslant 0, \quad u \not \equiv 0 & \text { in } \Omega,\end{cases}
$$

where $\Omega$ is an unbounded domain with smooth non-compact boundary $\Gamma, n$ denotes the unit outward normal vector on $\Gamma$, and $\lambda>0, \theta$ are real parameters. We assume throughout that $p<r<p^{*}=$ $p N /(N-p), 1<p<N$, the functions $a, b$ and $h$ are positive while $f, g$ are subcritical nonlinearities. We show that there exist an open interval $I$ and $\lambda^{\star}>0$ such that the problem has no solution if $\theta \in I$ and $\lambda \in\left(0, \lambda^{\star}\right)$. Furthermore, there exist an open interval $J \subset I$ and $\lambda_{0}>0$ such that, for any $\theta \in J$, the above problem has at least a solution if $\lambda \geqslant \lambda_{0}$, but it has no solution provided that $\lambda \in\left(0, \lambda_{0}\right)$.

Keywords: bifurcation problem; existence and non-existence results; weighted Sobolev spaces; anisotropic media

AMS 2000 Mathematics subject classification: Primary 35J60; 35P30; 58E05; 58G28

\section{Introduction}

Among the great range of processes modelled by nonlinear equations, those leading to bifurcation problems are of particular difficulty and importance. More precisely, many models from chemical engineering, mathematical biology, mechanics and engineering may be written in the form,

$$
u_{t}=\mathcal{F}\left(\lambda, u, D u, D^{2} u, \ldots\right) \quad \text { in } \Omega \times(0, T),
$$

where $u=u(x, t)$ is the state of the system under consideration. For instance, if we try to describe the behaviour of a bacteria culture, then the state variable $u$ might be the number of mass of the bacteria. In many concrete situations problems like (1.1) represent a complicated system of equations involving partial differential equations and other operations, like boundary or initial conditions. Each mathematical model contains (implicitly or explicitly) parameters corresponding to the real world situation being described. For example, the outcome of a bacteria-growing experiment will depend on the size of the 
experimental apparatus, the temperature, the composition of the ambient atmosphere, and other parameters. In such a way, a surprising variety of the problems in applied mathematics which exhibit multiple steady-state solutions, even systems with infinitely many degrees of freedom, can be reduced to the form,

$$
u_{t}=\mathcal{F}\left(\lambda_{1}, \lambda_{2}, \ldots, \lambda_{k}, u, D u, D^{2} u, \ldots\right) \quad \text { in } \Omega \times(0, T),
$$

which involves a large number $k$ of parameters. However, even for the biologists, it would be difficult to figure out how $\mathcal{F}$ should depend on all these quantities. In this case, in order to develop a consistent mathematical theory, one tries to fix as many as possible parameters and perhaps to vary one of them so as to see the effect of this. Several parameters in a model, such as Reynold's number, Lyapunov-Schmidt reduction, etc., can often be lumped into a single one by standard scaling procedures. Thus we obtain the evolution problem (1.1), which depends on a single parameter. The simplest solutions (1.1) can have are equilibrium solutions. These are time-independent solutions of (1.1), i.e. the states which satisfy $\mathcal{F}\left(\lambda, u, D u, D^{2} u, \ldots\right)=0$. Similar problems arise for the case of several state variables. We refer, for example, to the steady-state Brusselator model (see [4]), which was developed to describe morphogenesis and pattern formation in chemical reactions. We assume in this paper that $\mathcal{F}$ involves the quasilinear differential operator,

$$
\Delta_{p} u=\operatorname{div}\left(|D u|^{p-2} D u\right), \quad 1<p<\infty .
$$

We are concerned in this paper with the study of the following double bifurcation quasilinear problem,

$$
\left.\begin{array}{ll}
-\operatorname{div}\left(a(x)|D u|^{p-2} D u\right)+h(x) u^{r-1}=f(\lambda, x, u) & \text { in } \Omega \subset \mathbb{R}^{N}, \\
a(x)|D u|^{p-2} D u \cdot n+b(x) u^{p-1}=\theta g(x, u) & \text { on } \Gamma, \\
u \geqslant 0, \quad u \neq \equiv 0 & \text { in } \Omega,
\end{array}\right\}
$$

where $\Omega$ is an unbounded domain with non-compact, smooth boundary $\Gamma, \lambda>0, \theta$ are real parameters and throughout $p<r<p N /(N-p), 1<p<N$.

The study of non-trivial solutions in the above problem is motivated by the following example. Suppose an inviscid fluid flows irrotationally along a flat-bottomed canal. The flow can be modelled by an equation of the form $\mathcal{F}(\lambda, u, D u)=0$, where $\mathcal{F}(\lambda, 0,0)=0$. One possible motion is a uniform stream (corresponding to the trivial solution $u=0$ ), but it is of course the non-trivial solutions which are of physical interest.

Problems of this type arise in the study of physical phenomena related to equilibrium of anisotropic continuous media which possible are somewhere 'perfect' insulators (cf. [7]). For instance, if $\boldsymbol{\tau}$ denotes the shear stress and $D_{p} u$ is the velocity gradient then these quantities obey a relation of the form $\tau(x)=a(x) D_{p} u(x)$, where $D_{p} u=|D u|^{p-2} D u$. The case $p=2$ (respectively $p<2, p>2$ ) corresponds to a Newtonian (respectively pseudoplastic, dilatant) fluid. The resulting equations of motion then involve the quasilinear operator $\operatorname{div}\left(a D_{p} u\right)$. We refer in this sense to [2] for the mathematical treatment of the Hele-Shaw flow of 'power-law fluids'. The concept of Hele-Shaw flow refers to the flow between two closely spaced parallel plates, close in the sense that the gap between 
the plates is small compared to the dimension of the plates. Quasilinear problems with a variable coefficient also appear in the mathematical model of the torsional creep (elastic for $p=2$, plastic as $p \rightarrow \infty[\mathbf{3}, \mathbf{1 4}])$. This study is based on the observation that a prismatic material rod subject to a torsional moment, at sufficiently high temperature and for an extended period of time, exhibits a permanent deformation, called creep. The corresponding equations are derived under the assumptions that the components of strain and stress are linked by a power law referred to as the creep-law (see $[\mathbf{1 3}]$ and $[\mathbf{1 1}, \mathbf{1 2}]$ ). We also refer to the study of flow through porous media $(p=3 / 2[\mathbf{1 9}])$ or glacial sliding $\left(p \in\left(1, \frac{4}{3}\right][\mathbf{1 5}]\right)$. We mention the recent papers $[\mathbf{5}, \mathbf{9}, \mathbf{1 0}]$ for the mathematical treatment of bifurcation problems for several classes of quasilinear elliptic equations on unbounded domains and with respect to anisotropic spaces.

The purpose of this paper is to study a quasilinear eigenvalue problem with nonlinear boundary condition in an unbounded domain $\Omega \subset \mathbb{R}^{N}$, and we generalize in a larger framework some results from [6]. It is known that for unbounded domains, neither the embedding $W^{1, p}(\Omega) \hookrightarrow L^{q}(\Omega)$, nor the trace $W^{1, p}(\Omega) \rightarrow L^{m}(\Gamma)$ are compact. So, it is natural to look for more general function spaces, e.g. weighted Sobolev spaces, where compact embeddings can be obtained for suitable weight functions. However, because of the nonlinear boundary condition, it is not only necessary to have compact embeddings of weighted Sobolev spaces, but to also use compactness of the trace operator.

Pflüger [17] studied the trace operators $W^{1, p}\left(\Omega ; v_{0}, v_{1}\right) \rightarrow L^{s}(\Gamma ; w)$ in weighted Sobolev spaces for sufficiently regular unbounded domains $\Omega \subset \mathbb{R}^{N}$ with non-compact boundary. He established certain conditions on the weight functions $v_{0}, v_{1}, w$, which ensure the compactness of this operator.

For a positive measurable function $w_{1}$ defined in a domain $\Omega \subset \mathbb{R}^{N}$, let $L^{q}\left(\Omega ; w_{1}\right)$ be the space of all measurable functions $u$ such that

$$
\|u\|_{q, \Omega, w_{1}}=\left(\int_{\Omega}|u(x)|^{q} w_{1}(x) \mathrm{d} x\right)^{1 / q}
$$

is finite. If $\Gamma$ is a submanifold in $\mathbb{R}^{N}$, we denote by $L^{m}\left(\Gamma ; w_{2}\right)$ the space of all measurable functions $u$ such that $\|u\|_{m, \Gamma, w_{2}}$ is finite. The weighted Sobolev space $W^{1, p}\left(\Omega ; v_{0}, v_{1}\right)$ is defined as the set of all functions $u \in L^{p}\left(\Omega ; v_{0}\right)$ such that all the derivatives $u_{x_{i}}$ $(1 \leqslant i \leqslant N)$ belong to $L^{p}\left(\Omega ; v_{1}\right)$. The corresponding norm is given by

$$
\|u\|_{1, p, \Omega, v_{0}, v_{1}}=\left(\int_{\Omega}|u(x)|^{p} v_{0}(x) \mathrm{d} x+\int_{\Omega}|D u(x)|^{p} v_{1}(x) \mathrm{d} x\right)^{1 / p} .
$$

Denote by $A_{p}$ the Muckenhoupt class which is the set of all positive measurable functions $v$ in $\mathbb{R}^{N}$ satisfying

$$
\begin{aligned}
\frac{1}{|Q|}\left(\int_{Q} v \mathrm{~d} x\right)^{1 / p}\left(\int_{Q} v^{-1 /(p-1)} \mathrm{d} x\right)^{(p-1) / p} \leqslant C & \text { if } 1<p<\infty, \\
\frac{1}{|Q|} \int_{Q} v \mathrm{~d} x \leqslant C \underset{x \in Q}{\operatorname{sinf} \inf } v(x) & \text { if } p=1,
\end{aligned}
$$


for all cubes $Q$ in $\mathbb{R}^{N}$. For example, the function $v(x)=(1+|x|)^{\beta}$ belongs to $A_{p}$ if $\beta \in(-N, N(p-1))$ (see $[\mathbf{2 0}])$.

We always assume that the continuous weight functions $v_{0}, v_{1}, w_{0}, w_{1}, w_{2}$ belong to $A_{p}$. Furthermore, the unbounded domain $\Omega \subset \mathbb{R}^{N}$ and the weight functions are chosen such that we can apply $[\mathbf{1 7}$, Theorem 2] and $[\mathbf{1 7}$, Corollary 6] to guarantee that the trace $W^{1, p}\left(\Omega ; v_{0}, v_{1}\right) \rightarrow L^{p}\left(\Gamma ; w_{0}\right)$ is continuous and the embedding $W^{1, p}\left(\Omega ; v_{0}, v_{1}\right) \hookrightarrow$ $L^{q}\left(\Omega ; w_{1}\right)$ for some $p<q<p N /(N-p)$, respectively the trace $W^{1, p}\left(\Omega ; v_{0}, v_{1}\right) \rightarrow$ $L^{m}\left(\Gamma ; w_{2}\right)$ for some $p<m<p(N-1) /(N-p)$ are compact.

Remark 1.1. To give an example of the domain $\Omega \subset \mathbb{R}^{N}$ and of the weight functions $v_{0}, v_{1}, w_{0}, w_{1}$ and $w_{2}$ that satisfy the above assumptions, consider $\Omega$ as an infinite cylinder $\omega \times \mathbb{R}$ where $\omega \subset \mathbb{R}^{N-1}$ is smooth, bounded and

$$
\begin{aligned}
& v_{0}(x)=\frac{1}{(1+|x|)^{p}}, \quad v_{1}(x)=1, \\
& w_{0}(x)=(1+|x|)^{\alpha_{0}}, \quad w_{1}(x)=(1+|x|)^{\alpha_{1}}, \quad w_{2}(x)=(1+|x|)^{\alpha_{2}}, \quad x \in \mathbb{R}^{N} .
\end{aligned}
$$

To obtain continuity of the trace operator $W^{1, p}\left(\Omega ; v_{0}, v_{1}\right) \rightarrow L^{p}\left(\Gamma ; w_{0}\right)$ and compactness of the embedding $W^{1, p}\left(\Omega ; v_{0}, v_{1}\right) \hookrightarrow L^{q}\left(\Omega ; w_{1}\right)$ respectively of the trace operator $W^{1, p}\left(\Omega ; v_{0}, v_{1}\right) \rightarrow L^{m}\left(\Gamma ; w_{2}\right)$ we have to choose

$$
-N<\alpha_{0} \leqslant 1-p, \quad-N<\alpha_{1}<q \frac{N-p}{p}-N \quad \text { and } \quad-N<\alpha_{2}<m \frac{N-p}{p}-N+1 .
$$

Denote by $C_{\delta}^{\infty}(\Omega)$ the space of $C_{0}^{\infty}\left(\mathbb{R}^{N}\right)$-functions restricted to $\Omega$. We define the weighted Sobolev space $E$ as the completion of $C_{\delta}^{\infty}(\Omega)$ in the norm $\|\cdot\|_{E}$, where we shall use the abbreviation $\|\cdot\|_{E}=\|\cdot\|_{1, p, \Omega, v_{0}, v_{1}}$.

Remark 1.2. The definition of $E$ and the choice of our weight functions ensure the continuity of the trace $E \rightarrow L^{p}\left(\Gamma ; w_{0}\right)$ and the compactness of the embedding $E \hookrightarrow$ $L^{q}\left(\Omega ; w_{1}\right)$ respectively of the trace operator $E \rightarrow L^{m}\left(\Gamma ; w_{2}\right)$.

\section{Main results}

Suppose throughout this paper that the following hypotheses are fulfilled.

$\left(\mathbf{H}_{1}\right) v_{0} \in C^{1}\left(\mathbb{R}^{N}\right)$ and there exists a constant $0<\sigma<N$ such that

$$
|x| \cdot\left|D v_{0}(x)\right| \leqslant \sigma v_{0}(x) \quad \forall x \in \Omega ;
$$

$\left(\mathbf{H}_{2}\right) a$ is a positive measurable function, locally bounded in $\Omega$ and there exist positive constants $a_{0}, a_{1}$ such that

$$
a_{0}\left(|x|^{p} v_{0}(x)+v_{1}(x)\right) \leqslant a(x) \leqslant a_{1} v_{1}(x) \quad \text { a.e. } x \in \Omega ;
$$

$\left(\mathbf{H}_{\mathbf{3}}\right) b$ is a positive continuous function on $\mathbb{R}^{N}$ and there exist positive constants $b_{0}$ and $b_{1}$ such that

$$
b_{0}|x| v_{0}(x) \leqslant b(x) \leqslant b_{1} w_{0}(x) \quad \text { a.e. } x \in \Gamma \text {. }
$$


Let $f(\lambda, x, s):(0, \infty) \times \Omega \times \mathbb{R} \rightarrow \mathbb{R}$ be non-decreasing in $\lambda$, measurable in $x$, derivable in $s$ satisfying

$\left(\mathbf{H}_{4}\right) f(\cdot, \cdot, 0)=0, f(\lambda, x, s)+f(\lambda, x,-s) \geqslant 0 \forall \lambda>0$, a.e. $x \in \Omega, \forall s \in \mathbb{R}$;

$\left(\mathbf{H}_{\mathbf{5}}\right)\left|f_{s}(\lambda, x, s)\right| \leqslant \lambda \varphi(x)|s|^{q-2}$ for some $p<q<r, \forall \lambda>0$, a.e. $x \in \Omega, \forall s \in \mathbb{R}$, where $\varphi$ is a non-negative, measurable function such that

$$
0 \leqslant \varphi(x) \leqslant c_{f} w_{1}(x) \quad \text { a.e. } x \in \Omega
$$

$\left(\mathbf{H}_{\mathbf{6}}\right) \lim _{s \rightarrow 0} \frac{f(\lambda, x, s)}{\lambda w_{1}(x)|s|^{q-2} s}=1$ uniformly in $x$ and in $\lambda$;

$\left(\mathbf{H}_{7}\right)\left|f\left(\lambda_{1}, x, s\right)-f\left(\lambda_{2}, x, s\right)\right| \leqslant\left|\lambda_{1}-\lambda_{2}\right| \psi(x)|s|^{q-1}, \forall \lambda_{1}, \lambda_{2}>0$, a.e. $x \in \Omega, \forall s \in \mathbb{R}$, where $\psi$ is a non-negative, measurable function such that

$$
0 \leqslant \psi(x) \leqslant C_{f} w_{1}(x) \quad \text { a.e. } x \in \Omega .
$$

Assume $g: \Gamma \times \mathbb{R} \rightarrow \mathbb{R}$ is a Carathéodory function that satisfies the following conditions.

$\left(\mathbf{H}_{\mathbf{8}}\right) g(\cdot, 0)=0, g(x, s)+g(x,-s) \geqslant 0$ a.e. $x \in \Gamma, \forall s \in \mathbb{R}$;

$\left(\mathbf{H}_{\mathbf{9}}\right)|g(x, s)| \leqslant g_{0}(x)+g_{1}(x)|s|^{m-1}$, for some $p<m<p(N-1) /(N-p)$, a.e. $x \in$ $\Omega, \forall s \in \mathbb{R}$, where $g_{0}, g_{1}$ are non-negative, measurable functions such that

$$
0 \leqslant g_{0}(x), \quad g_{1}(x) \leqslant C_{g} w_{2}(x) \quad \text { a.e. } x \in \Gamma, \quad g_{0} \in L^{m /(m-1)}\left(\Gamma ; w_{2}^{1 /(1-m)}\right) .
$$

The following integrability condition of the ratio $w_{1}^{r} / h^{q}$ is inspired by assumption (1.4) in $[\mathbf{1}]$.

$\left(\mathbf{H}_{10}\right) h: \Omega \rightarrow \mathbb{R}$ is a positive and continuous function satisfying

$$
\int_{\Omega}\left(\frac{w_{1}^{r}}{h^{q}}\right)^{1 /(r-q)} \mathrm{d} x<\infty .
$$

Remark 2.1. If $0<\underline{a} \leqslant a \in L^{\infty}(\Omega)$ and $b \in C\left(\mathbb{R}^{N}\right)$ is a positive function such that

$$
\frac{c_{1}}{(1+|x|)^{p-1}} \leqslant b(x) \leqslant \frac{c_{2}}{(1+|x|)^{p-1}} \quad \text { for some constants } 0<c_{1} \leqslant c_{2},
$$

then hypotheses $\left(\mathbf{H}_{\mathbf{1}}\right)-\left(\mathbf{H}_{\mathbf{3}}\right)$ are fulfilled if we take weight functions as in Remark 1.1 with $\alpha_{0}=1-p$.

Consider the Banach space $X=E \cap L^{r}(\Omega ; h)$ endowed with the norm,

$$
\|u\|_{X}^{p}:=\|u\|_{E}^{p}+\left(\int_{\Omega}|u(x)|^{r} h(x) \mathrm{d} x\right)^{p / r} .
$$


Obviously, the following embeddings

$$
X \stackrel{i}{\hookrightarrow} E \quad \text { and } \quad X \stackrel{j}{\hookrightarrow} L^{r}(\Omega ; h) \quad \text { are continuous. }
$$

The energy functional corresponding to $\left(\mathrm{P}_{\lambda, \theta}\right)$ is given by $\Phi_{\lambda, \theta}: X \rightarrow \mathbb{R}$,

$$
\begin{array}{r}
\Phi_{\lambda, \theta}(u)=\frac{1}{p} \int_{\Omega} a(x)|D u|^{p} \mathrm{~d} x+\frac{1}{p} \int_{\Gamma} b(x)|u|^{p} \mathrm{~d} \Gamma+\frac{1}{r} \int_{\Omega} h(x)|u|^{r} \mathrm{~d} x \\
-\int_{\Omega} F(\lambda, x, u) \mathrm{d} x-\theta \int_{\Gamma} G(x, u) \mathrm{d} \Gamma,
\end{array}
$$

where $F$ and $G$ denote the primitive functions of $f$ and $g$ with respect to the last variable, i.e.

$$
F(\lambda, x, u)=\int_{0}^{u} f(\lambda, x, s) \mathrm{d} s, \quad G(x, u)=\int_{0}^{u} g(x, s) \mathrm{d} s .
$$

Solutions to problem $\left(\mathrm{P}_{\lambda, \theta}\right)$ will be found as non-negative and non-trivial critical points of $\Phi_{\lambda, \theta}$. Therefore, a function $u \in X$ is a solution of the problem $\left(\mathrm{P}_{\lambda, \theta}\right)$ provided that $u \geqslant 0, u \neq \equiv$ in $\Omega$ and for any $v \in X$,

$$
\begin{aligned}
\int_{\Omega} a(x)|D u|^{p-2} D u & \cdot D v \mathrm{~d} x+\int_{\Gamma} b(x)|u|^{p-2} u v \mathrm{~d} \Gamma \\
& +\int_{\Omega} h(x)|u|^{r-2} u v \mathrm{~d} x-\theta \int_{\Gamma} g(x, u) v \mathrm{~d} \Gamma=\int_{\Omega} f(\lambda, x, u) v \mathrm{~d} x .
\end{aligned}
$$

Set

$$
\begin{aligned}
\mathcal{N}_{g}:=\left\{u \in X: \int_{\Gamma} g(x, u) u \mathrm{~d} \Gamma<0\right\}, & \mathcal{P}_{g}:=\left\{u \in X: \int_{\Gamma} g(x, u) u \mathrm{~d} \Gamma>0\right\} \\
\theta_{*}:=\sup _{u \in \mathcal{N}_{g}} \frac{\|u\|_{b}^{p}}{\int_{\Gamma} g(x, u) u \mathrm{~d} \Gamma}, & \theta^{*}:=\inf _{u \in \mathcal{P}_{g}} \frac{\|u\|_{b}^{p}}{\int_{\Gamma} g(x, u) u \mathrm{~d} \Gamma},
\end{aligned}
$$

where $\|\cdot\|_{b}$ is defined on $E$ as follows:

$$
\|u\|_{b}:=\left(\int_{\Omega} a(x)|D u|^{p} \mathrm{~d} x+\int_{\Gamma} b(x)|u|^{p} \mathrm{~d} \Gamma\right)^{1 / p} .
$$

We introduce the convention that if $\mathcal{N}_{g}=\emptyset$ then $\theta_{*}=-\infty$ and $\theta^{*}=+\infty$, provided $\mathcal{P}_{g}=\emptyset$. Define

$$
\begin{aligned}
\mathcal{N}_{G}:=\left\{u \in X: \int_{\Gamma} G(x, u) \mathrm{d} \Gamma<0\right\}, & \mathcal{P}_{G}:=\left\{u \in X: \int_{\Gamma} G(x, u) \mathrm{d} \Gamma>0\right\} \\
\theta_{-}:=\sup _{u \in \mathcal{N}_{G}} \frac{\|u\|_{b}^{p}}{p \int_{\Gamma} G(x, u) \mathrm{d} \Gamma}, & \theta^{+}:=\inf _{u \in \mathcal{P}_{G}} \frac{\|u\|_{b}^{p}}{p \int_{\Gamma} G(x, u) \mathrm{d} \Gamma} .
\end{aligned}
$$

If $\mathcal{N}_{G}=\emptyset$ (respectively, $\left.\mathcal{P}_{G}=\emptyset\right)$ then we set $\theta_{-}=-\infty$ (respectively, $\left.\theta^{+}=+\infty\right)$.

Our main results are the following. 
Theorem 2.2. Suppose $\theta_{*}<\theta<\theta^{*}$. Then there exists $\lambda^{*}>0$ such that problem $\left(\mathrm{P}_{\lambda, \theta}\right)$ has no solution, provided that $0<\lambda<\lambda^{*}$.

In order to state the next result, let us denote $\underline{\theta}=\max \left\{\theta_{*}, \theta_{-}\right\}$and $\bar{\theta}=\min \left\{\theta^{*}, \theta^{+}\right\}$. We observe that $\underline{\theta} \leqslant 0 \leqslant \bar{\theta}$. Set $J=(\underline{\theta}, \bar{\theta})$ and assume that $J \neq \emptyset$.

Theorem 2.3. Suppose $\theta \in J$. Then there exists $\lambda_{0}>0$ such that the following hold:

(i) problem $\left(\mathrm{P}_{\lambda, \theta}\right)$ admits a solution, for any $\lambda \geqslant \lambda_{0}$;

(ii) problem $\left(\mathrm{P}_{\lambda, \theta}\right)$ does not have any solution, provided that $0<\lambda<\lambda_{0}$.

\section{Auxiliary results}

We first prove that the energy functional $\Phi_{\lambda, \theta}$ is well defined on $X$.

Lemma 3.1. There exist positive constants $C_{1}$ and $C_{2}$ such that for every $u \in E$

$$
\int_{\Omega}|u|^{p} v_{0}(x) \mathrm{d} x \leqslant C_{1} \int_{\Omega}|D u|^{p} v_{1}(x) \mathrm{d} x+C_{2} \int_{\Gamma}|n \cdot x||u|^{p} v_{0}(x) \mathrm{d} \Gamma .
$$

Proof. Using the divergence theorem we obtain, for any $u \in C_{\delta}^{\infty}(\Omega)$,

$$
\int_{\Omega} x \cdot D\left(|u|^{p} v_{0}(x)\right) \mathrm{d} x=\int_{\Gamma}(n \cdot x)|u|^{p} v_{0}(x) \mathrm{d} \Gamma-N \int_{\Omega}|u|^{p} v_{0}(x) \mathrm{d} x .
$$

This implies

$$
\begin{aligned}
N \int_{\Omega}|u|^{p} v_{0}(x) \mathrm{d} x \leqslant \int_{\Gamma} \mid n \cdot & x \|\left.\right|^{p} v_{0}(x) \mathrm{d} \Gamma \\
& \quad+\int_{\Omega}|u|^{p}|x|\left|D v_{0}(x)\right| \mathrm{d} x+p \int_{\Omega}|x||u|^{p-1}|D u| v_{0}(x) \mathrm{d} x .
\end{aligned}
$$

Using Hölder's and Young's inequality, we get the estimate

$$
\begin{aligned}
p \int_{\Omega}|x||u|^{p-1}|D u| v_{0}(x) \mathrm{d} x & \leqslant p\left(\int_{\Omega}|u|^{p} v_{0}(x) \mathrm{d} x\right)^{(p-1) / p}\left(\int_{\Omega}|D u|^{p}|x|^{p} v_{0}(x) \mathrm{d} x\right)^{1 / p} \\
& \leqslant \varepsilon(p-1) \int_{\Omega}|u|^{p} v_{0}(x) \mathrm{d} x+\varepsilon^{1-p} \int_{\Omega}|D u|^{p}|x|^{p} v_{0} \mathrm{~d} x
\end{aligned}
$$

where $\varepsilon>0$ is an arbitrary real number. From (3.1), (3.2) and $\left(\mathbf{H}_{\mathbf{1}}\right)$ it follows that

$$
(N-\varepsilon(p-1)-\sigma) \int_{\Omega}|u|^{p} v_{0}(x) \mathrm{d} x \leqslant \varepsilon^{1-p} \int_{\Omega}|D u|^{p}|x|^{p} v_{0}(x) \mathrm{d} x+\int_{\Gamma}|n \cdot x||u|^{p} v_{0}(x) \mathrm{d} \Gamma .
$$

Using $\left(\mathbf{H}_{\mathbf{2}}\right)$ and choosing $\varepsilon$ small enough we find

$$
\int_{\Omega}|u|^{p} v_{0}(x) \mathrm{d} x \leqslant C_{1} \int_{\Omega}|D u|^{p} v_{1}(x) \mathrm{d} x+C_{2} \int_{\Gamma}|n \cdot x||u|^{p} v_{0}(x) \mathrm{d} \Gamma, \quad \forall u \in C_{\delta}^{\infty}(\Omega) .
$$

The conclusion of our lemma follows now by standard density arguments. 
Lemma 3.2. The quantity $\|\cdot\|_{b}$ defined by (2.2) represents an equivalent norm on $E$.

Proof. The inequality $\|u\|_{E}^{p} \leqslant c\|u\|_{b}^{p}$ follows directly from Lemma 3.1 by using the left hand side inequalities which appear in hypotheses $\left(\mathbf{H}_{\mathbf{2}}\right)$ and $\left(\mathbf{H}_{\mathbf{3}}\right)$.

By Remark 1.2 we know that the trace $E \rightarrow L^{p}\left(\Gamma ; w_{0}\right)$ is continuous. Therefore, we have that there exists $C>0$ such that

$$
\int_{\Gamma}|u|^{p} w_{0}(x) \mathrm{d} \Gamma \leqslant C\|u\|_{E}^{p} \quad \forall u \in E .
$$

Using the inequalities remained in $\left(\mathbf{H}_{\mathbf{2}}\right),\left(\mathbf{H}_{\mathbf{3}}\right)$ and by (3.3) it follows that

$$
\|u\|_{b}^{p} \leqslant a_{1} \int_{\Omega}|D u|^{p} v_{1}(x) \mathrm{d} x+b_{1} \int_{\Gamma}|u|^{p} w_{0}(x) \mathrm{d} \Gamma \leqslant c^{\prime}\|u\|_{E}^{p} .
$$

Hence the desired equivalence is proved.

For $\lambda>0$ fixed, let $f_{\lambda}$ be the function defined by

$$
f_{\lambda}(x, s)=f(\lambda, x, s) \quad \forall x \in \Omega, \forall s \in \mathbb{R} .
$$

Set $F_{\lambda}(x, u)=\int_{0}^{u} f_{\lambda}(x, s) \mathrm{d} s$. Denote by $N_{f_{\lambda}}, N_{F_{\lambda}}, N_{g}, N_{G}$ the corresponding Nemytskii operators.

Lemma 3.3. The operators,

$$
\begin{array}{rlrl}
N_{f_{\lambda}}: L^{q}\left(\Omega ; w_{1}\right) & \rightarrow L^{q /(q-1)}\left(\Omega ; w_{1}^{1 /(1-q)}\right), & & N_{F_{\lambda}}: L^{q}\left(\Omega ; w_{1}\right) \rightarrow L^{1}(\Omega), \\
N_{g}: L^{m}\left(\Gamma ; w_{2}\right) \rightarrow L^{m /(m-1)}\left(\Gamma ; w_{2}^{1 /(1-m)}\right), & & N_{G}: L^{m}\left(\Gamma ; w_{2}\right) \rightarrow L^{1}(\Gamma),
\end{array}
$$

are bounded and continuous.

Proof. From hypothesis $\left(\mathbf{H}_{\mathbf{5}}\right)$ we deduce that

$$
\left.\begin{array}{ll}
\left|f_{\lambda}(x, u)\right| \leqslant \frac{\lambda}{q-1} \varphi(x)|u|^{q-1} \leqslant \tilde{C}_{f} \lambda|u|^{q-1} w_{1}(x) & \text { a.e. } x \in \Omega, \forall u \in \mathbb{R}, \\
\left|F_{\lambda}(x, u)\right| \leqslant \frac{\lambda}{q(q-1)} \varphi(x)|u|^{q} \leqslant \frac{\tilde{C}_{f}}{q} \lambda|u|^{q} w_{1}(x) & \text { a.e. } x \in \Omega, \forall u \in \mathbb{R},
\end{array}\right\}
$$

where $\tilde{C}_{f}$ denotes $c_{f} /(q-1)$.

For $u \in L^{q}\left(\Omega ; w_{1}\right)$ we get (setting $q^{\prime}=q /(q-1)$ )

$$
\int_{\Omega}\left|N_{f_{\lambda}}(u)\right|^{q^{\prime}} w_{1}^{1 /(1-q)} \mathrm{d} x \leqslant\left(\tilde{C}_{f} \lambda\right)^{q^{\prime}} \int_{\Omega}|u|^{q} w_{1}(x) \mathrm{d} x .
$$

Therefore, $N_{f_{\lambda}}$ is bounded. Similarly, the boundedness of $N_{F_{\lambda}}$ follows from the estimate

$$
\int_{\Omega}\left|N_{F_{\lambda}}(u)\right| \mathrm{d} x \leqslant \frac{\tilde{C}_{f}}{q} \lambda \int_{\Omega}|u|^{q} w_{1}(x) \mathrm{d} x .
$$


Let $m^{\prime}=m /(m-1)$ and $u \in L^{m}\left(\Gamma ; w_{2}\right)$. Then, by $\left(\mathbf{H}_{\mathbf{9}}\right)$

$$
\begin{aligned}
\int_{\Gamma}\left|N_{g}(u)\right|^{m^{\prime}} w_{2}^{1 /(1-m)} \mathrm{d} \Gamma & \leqslant 2^{m^{\prime}-1}\left(\int_{\Gamma} g_{0}^{m^{\prime}} w_{2}^{1 /(1-m)} \mathrm{d} \Gamma+\int_{\Gamma} g_{1}^{m^{\prime}}|u|^{m} w_{2}^{1 /(1-m)} \mathrm{d} \Gamma\right) \\
& \leqslant 2^{m^{\prime}-1}\left(C+C_{g}^{m^{\prime}} \int_{\Gamma}|u|^{m} w_{2}(x) \mathrm{d} \Gamma\right),
\end{aligned}
$$

which shows that $N_{g}$ is bounded. In a similar way, by $\left(\mathbf{H}_{\mathbf{9}}\right)$ and Hölder's inequality we obtain

$$
\begin{aligned}
& \int_{\Gamma}\left|N_{G}(u)\right| \mathrm{d} \Gamma \\
& \leqslant \int_{\Gamma} g_{0}|u| \mathrm{d} \Gamma+\frac{1}{m} \int_{\Gamma} g_{1}|u|^{m} \mathrm{~d} \Gamma \\
& \leqslant\left(\int_{\Gamma} g_{0}^{m^{\prime}} w_{2}^{1 /(1-m)} \mathrm{d} \Gamma\right)^{1 / m^{\prime}} \cdot\left(\int_{\Gamma}|u|^{m} w_{2}(x) \mathrm{d} \Gamma\right)^{1 / m}+\frac{C_{g}}{m} \int_{\Gamma}|u|^{m} w_{2}(x) \mathrm{d} \Gamma,
\end{aligned}
$$

and the boundedness of $N_{G}$ follows.

From the usual properties of Nemytskii operators we deduce the continuity of $N_{f_{\lambda}}$, $N_{F_{\lambda}}, N_{g}$ and $N_{G}($ see $[\mathbf{2 1}])$.

In view of Lemmas 3.2 and $3.3, \Phi_{\lambda, \theta}$ is well defined on $X$.

Lemma 3.4. The functional $\Phi_{\lambda, \theta}$ is Fréchet-differentiable on $X$.

Proof. We use the notation,

$$
\begin{aligned}
I(u) & =\frac{1}{p}\|u\|_{b}^{p}, & J(u) & =\frac{1}{r}\|u\|_{r, \Omega, h}^{r}, \\
K_{G}(u) & =\int_{\Gamma} G(x, u) \mathrm{d} \Gamma, & K_{F_{\lambda}}(u) & =\int_{\Omega} F_{\lambda}(x, u) \mathrm{d} x .
\end{aligned}
$$

Then the Gâteaux derivative of $\Phi_{\lambda, \theta}$ is given by

$$
\left\langle\Phi_{\lambda, \theta}^{\prime}(u), v\right\rangle=\left\langle I^{\prime}(u), v\right\rangle+\left\langle J^{\prime}(u), v\right\rangle-\left\langle K_{F_{\lambda}}^{\prime}(u), v\right\rangle-\theta\left\langle K_{G}^{\prime}(u), v\right\rangle,
$$

where

$$
\begin{aligned}
\left\langle I^{\prime}(u), v\right\rangle & =\int_{\Omega} a(x)|D u|^{p-2} D u \cdot D v \mathrm{~d} x+\int_{\Gamma} b(x)|u|^{p-2} u v \mathrm{~d} \Gamma, \\
\left\langle J^{\prime}(u), v\right\rangle & =\int_{\Omega} h(x)|u|^{r-2} u v \mathrm{~d} x, \\
\left\langle K_{F_{\lambda}}^{\prime}(u), v\right\rangle & =\int_{\Omega} f_{\lambda}(x, u) v \mathrm{~d} x \\
\left\langle K_{G}^{\prime}(u), v\right\rangle & =\int_{\Gamma} g(x, u) v \mathrm{~d} \Gamma .
\end{aligned}
$$

We need only to show the continuity of $\Phi_{\lambda, \theta}^{\prime}$ and the assertion is proved. 
Clearly, $I^{\prime}: E \rightarrow E^{\prime}$ and $J^{\prime}: L^{r}(\Omega ; h) \rightarrow\left(L^{r}(\Omega ; h)\right)^{\prime}$ are continuous. By using (2.1) we see immediately that $I^{\prime}: X \rightarrow X^{\prime}$ and $J^{\prime}: X \rightarrow X^{\prime}$ are continuous.

The operator $K_{G}^{\prime}$ is a composition of operators

$$
X \stackrel{i}{\hookrightarrow} E \stackrel{\gamma}{\rightarrow} L^{m}\left(\Gamma ; w_{2}\right) \stackrel{N_{g}}{\rightarrow} L^{m /(m-1)}\left(\Gamma ; w_{2}^{1 /(1-m)}\right) \stackrel{k}{\rightarrow} E^{\prime} \stackrel{i^{\prime}}{\hookrightarrow} X^{\prime},
$$

where $\langle k(u), v\rangle=\int_{\Gamma} u v \mathrm{~d} \Gamma$. Obviously, $k$ is a linear operator. By Hölder's inequality and Remark 1.2,

$$
\begin{aligned}
\int_{\Gamma}|u v| \mathrm{d} \Gamma & \leqslant\left(\int_{\Gamma}|u|^{m^{\prime}} w_{2}^{1 /(1-m)} \mathrm{d} \Gamma\right)^{1 / m^{\prime}} \cdot\left(\int_{\Gamma}|v|^{m} w_{2} \mathrm{~d} \Gamma\right)^{1 / m} \\
& \leqslant C\|u\|_{m /(m-1), \Gamma, w_{2}^{1 /(1-m)}}\|v\|_{E}
\end{aligned}
$$

which shows that $k$ is continuous. As a composition of continuous operators, $K_{G}^{\prime}$ is continuous, too. Moreover, it is compact since the trace operator $\gamma$ is compact. In a similar way we obtain that $K_{F_{\lambda}}^{\prime}$ is continuous such that the Fréchet-differentiability of $\Phi_{\lambda, \theta}$ follows.

\section{Proof of Theorem 2.2}

Assume $\theta_{*}<\theta<\theta^{*}$ and let $\lambda>0$ be chosen such that problem $\left(\mathrm{P}_{\lambda, \theta}\right)$ possesses at least a solution. We claim that there exists $\lambda^{*}>0$ such that $\lambda \geqslant \lambda^{*}$. Suppose that $u$ is a solution of problem $\left(\mathrm{P}_{\lambda, \theta}\right)$. Then, using (3.4) we find

$$
\begin{aligned}
\|u\|_{b}^{p}-\theta \int_{\Gamma} g(x, u) u \mathrm{~d} \Gamma+\int_{\Omega} h(x)|u|^{r} \mathrm{~d} x & =\int_{\Omega} f(\lambda, x, u) u \mathrm{~d} x \\
& \leqslant \lambda \tilde{C}_{f} \int_{\Omega}|u|^{q} w_{1}(x) \mathrm{d} x .
\end{aligned}
$$

Now, Young's inequality implies the following estimate:

$$
\begin{aligned}
\lambda \tilde{C}_{f} \int_{\Omega}|u|^{q} w_{1}(x) \mathrm{d} x & =\int_{\Omega} \frac{\lambda \tilde{C}_{f} w_{1}}{h^{q / r}} \cdot h^{q / r}|u|^{q} \mathrm{~d} x \\
& \leqslant \frac{r-q}{r}\left(\tilde{C}_{f} \lambda\right)^{r /(r-q)} \int_{\Omega}\left(\frac{w_{1}^{r}}{h^{q}}\right)^{1 /(r-q)} \mathrm{d} x+\frac{q}{r} \int_{\Omega} h|u|^{r} \mathrm{~d} x .
\end{aligned}
$$

This inequality combined with (4.1) gives

$$
\begin{aligned}
\|u\|_{b}^{p}-\theta \int_{\Gamma} g(x, u) u \mathrm{~d} \Gamma & \leqslant \frac{r-q}{r}\left(\tilde{C}_{f} \lambda\right)^{r /(r-q)} \int_{\Omega}\left(\frac{w_{1}^{r}}{h^{q}}\right)^{1 /(r-q)} \mathrm{d} x+\frac{q-r}{r} \int_{\Omega} h|u|^{r} \mathrm{~d} x \\
& \leqslant \frac{r-q}{r}\left(\tilde{C}_{f} \lambda\right)^{r /(r-q)} \int_{\Omega}\left(\frac{w_{1}^{r}}{h^{q}}\right)^{1 /(r-q)} \mathrm{d} x .
\end{aligned}
$$

On the one hand, $\theta<\theta^{*}$ implies the existence of a constant $C_{1} \in(0,1)$ such that

$$
\theta \leqslant\left(1-C_{1}\right) \theta^{*} \leqslant\left(1-C_{1}\right) \frac{\|u\|_{b}^{p}}{\int_{\Gamma} g(x, u) u \mathrm{~d} \Gamma} \quad \text { for all } u \in \mathcal{P}_{g}
$$


which yields

$$
\|u\|_{b}^{p}-\theta \int_{\Gamma} g(x, u) u \mathrm{~d} \Gamma \geqslant C_{1}\|u\|_{b}^{p} \quad \text { for all } u \in \mathcal{P}_{g} .
$$

On the other hand, $\theta_{*}<\theta$ shows that there exists $C_{2} \in(0,1)$ such that

$$
\|u\|_{b}^{p}-\theta \int_{\Gamma} g(x, u) u \mathrm{~d} \Gamma \geqslant C_{2}\|u\|_{b}^{p} \quad \text { for all } u \in \mathcal{N}_{g} .
$$

From (4.3) and (4.4) we conclude that

$$
\|u\|_{b}^{p}-\theta \int_{\Gamma} g(x, u) u \mathrm{~d} \Gamma \geqslant C_{0}\|u\|_{b}^{p} \quad \text { for all } u \in X
$$

where $C_{0}=\min \left\{C_{1}, C_{2}\right\}$.

The continuity of the embedding $E \hookrightarrow L^{q}\left(\Omega ; w_{1}\right)$ implies the existence of $\bar{C}>0$ such that

$$
\bar{C}\|u\|_{q, \Omega, w_{1}}^{p} \leqslant\|u\|_{b}^{p} \quad \text { for all } u \in E .
$$

By (4.1) and (4.5) we have

$$
C_{0} \bar{C}\left(\int_{\Omega}|u|^{q} w_{1}(x) \mathrm{d} x\right)^{p / q} \leqslant C_{0}\|u\|_{b}^{p} \leqslant \lambda \tilde{C}_{f} \int_{\Omega}|u|^{q} w_{1}(x) \mathrm{d} x
$$

which implies

$$
\left(\bar{C} C_{0} \tilde{C}_{f}^{-1} \lambda^{-1}\right)^{q /(q-p)} \leqslant \int_{\Omega}|u|^{q} w_{1}(x) \mathrm{d} x .
$$

This combined with (4.6) yields

$$
C_{0} \bar{C}\left(\bar{C} C_{0} \tilde{C}_{f}^{-1} \lambda^{-1}\right)^{p /(q-p)} \leqslant C_{0}\|u\|_{b}^{p} .
$$

Using (4.7) together with (4.2) and (4.5) we obtain

$$
C_{0} \bar{C}\left(\bar{C} C_{0} \tilde{C}_{f}^{-1} \lambda^{-1}\right)^{p /(q-p)} \leqslant \frac{r-q}{r}\left(\tilde{C}_{f} \lambda\right)^{r /(r-q)} \int_{\Omega}\left(\frac{w_{1}^{r}}{h^{q}}\right)^{1 /(r-q)} \mathrm{d} x .
$$

We see that our claim follows if we take

$$
\lambda^{*}=C^{*}\left(\int_{\Omega}\left(\frac{w_{1}^{r}}{h^{q}}\right)^{1 /(r-q)} \mathrm{d} x\right)^{-(q-p)(r-q) / q(r-p)},
$$

where $C^{*}$ denotes

$$
\tilde{C}_{f}^{-1}\left[C_{0} \bar{C}\left(\frac{r}{r-q}\right)^{(q-p) / q}\right]^{(r-q) /(r-p)}
$$


Corollary 4.1. Suppose $\theta_{*}<\theta<\theta^{*}$ and $\lambda>0$ such that $\left(\mathrm{P}_{\lambda, \theta}\right)$ has a solution $u$. Then

$$
C_{0}\|u\|_{b}^{p}+\frac{r-q}{r} \int_{\Omega} h|u|^{r} \mathrm{~d} x \leqslant \frac{r-q}{r}\left(\tilde{C}_{f} \lambda\right)^{r /(r-q)} \int_{\Omega}\left(\frac{w_{1}^{r}}{h^{q}}\right)^{1 /(r-q)} \mathrm{d} x
$$

and

$$
\|u\|_{b} \geqslant K \lambda^{-1 /(q-p)}
$$

where $K>0$ is a constant independent of $u$.

Proof. The first part of the assertion follows by (4.2) and (4.5). The second one is implied by (4.7), which shows that the constant $K$ can be chosen, for example, as $\bar{C}^{q / p(q-p)}\left(C_{0} \tilde{C}_{f}^{-1}\right)^{1 /(q-p)}$.

\section{Properties of $\Phi_{\lambda, \theta}$}

Proceeding in the same manner as we did for proving (4.5), we can show that if we take $\theta_{-}<\theta<\theta^{+}$, then there exists $c>0$ such that

$$
\frac{1}{p}\|u\|_{b}^{p}-\theta \int_{\Gamma} G(x, u) \mathrm{d} \Gamma \geqslant c\|u\|_{b}^{p} \quad \text { for all } u \in X .
$$

We shall employ in what follows the following elementary inequality:

$$
s|u|^{\mu}-t|u|^{\nu} \leqslant C_{\mu, \nu} s\left(\frac{s}{t}\right)^{\mu /(\nu-\mu)} \quad \forall u \in \mathbb{R}, \forall s, t \in(0, \infty), \forall 0<\mu<\nu .
$$

Lemma 5.1. Suppose $\theta_{-}<\theta<\theta^{+}$and $\lambda>0$ is arbitrary. Then the functional $\Phi_{\lambda, \theta}$ is coercive.

Proof. From (3.4) we have that there exists $C>0$ such that

$$
F(\lambda, x, u) \leqslant C \lambda|u|^{q} w_{1}(x) \quad \text { a.e. } x \in \Omega, \forall u \in \mathbb{R} .
$$

By virtue of (5.2) and $\left(\mathbf{H}_{\mathbf{1 0}}\right)$ we obtain

$$
\begin{aligned}
\int_{\Omega}\left(C \lambda w_{1}|u|^{q}-\frac{h}{2 r}|u|^{r}\right) \mathrm{d} x & \leqslant C_{r, q} \int_{\Omega} \lambda w_{1}\left(\frac{\lambda w_{1}}{h}\right)^{q /(r-q)} \mathrm{d} x \\
& =C_{r, q} \lambda^{r /(r-q)} \int_{\Omega}\left(\frac{w_{1}^{r}}{h^{q}}\right)^{1 /(r-q)} \mathrm{d} x \leqslant C^{\prime} .
\end{aligned}
$$

Using (5.1), (5.3) and the above estimate we find

$$
\begin{aligned}
\Phi_{\lambda, \theta}(u) & =\frac{1}{p}\|u\|_{b}^{p}-\theta \int_{\Gamma} G(x, u) \mathrm{d} \Gamma-\int_{\Omega} F(\lambda, x, u) \mathrm{d} x+\frac{1}{r} \int_{\Omega} h|u|^{r} \mathrm{~d} x \\
& \geqslant c\|u\|_{b}^{p}-\int_{\Omega}\left(C \lambda|u|^{q} w_{1}-\frac{h}{2 r}|u|^{r}\right) \mathrm{d} x+\frac{1}{2 r} \int_{\Omega} h|u|^{r} \mathrm{~d} x \\
& \geqslant c\|u\|_{b}^{p}+\frac{1}{2 r} \int_{\Omega} h|u|^{r} \mathrm{~d} x-C^{\prime}
\end{aligned}
$$

and the coercivity of $\Phi_{\lambda, \theta}$ follows. 
Lemma 5.2. Suppose $\theta_{-}<\theta<\theta^{+}, \lambda>0$ is arbitrary and $\left\{u_{n}\right\}$ is a sequence in $X$ such that $\Phi_{\lambda, \theta}\left(u_{n}\right)$ is bounded. Then there exists a subsequence of $\left\{u_{n}\right\}$, denoted again by $\left\{u_{n}\right\}$, such that

$$
u_{n} \rightarrow u_{0} \text { in } X, \quad u_{n} \rightarrow u_{0} \text { a.e. in } \Omega \quad \text { and } \quad \Phi_{\lambda, \theta}\left(u_{0}\right) \leqslant \liminf _{n \rightarrow \infty} \Phi_{\lambda, \theta}\left(u_{n}\right) .
$$

Proof. In view of Lemma 5.1, the boundedness of $\Phi_{\lambda, \theta}\left(u_{n}\right)$ shows that $\left\{u_{n}\right\}$ must be bounded in $X$. Using (2.1) and Remark 1.2 we may assume (up to a subsequence) that

$$
u_{n} \rightarrow u_{0} \text { in } X, \quad u_{n} \rightarrow u_{0} \text { in } L^{q}\left(\Omega ; w_{1}\right) \quad \text { and } \quad u_{n} \rightarrow u_{0} \text { a.e. in } \Omega .
$$

Set

$$
\Xi(x, u)=F(\lambda, x, u)-\frac{1}{r} h|u|^{r} \quad \text { and } \quad \xi(x, u)=\Xi_{u}(x, u) .
$$

By hypothesis $\left(\mathbf{H}_{\mathbf{5}}\right)$ and $(5.2)$ we obtain

$$
\begin{aligned}
\xi_{u}(x, u) & =f_{u}(\lambda, x, u)-(r-1) h|u|^{r-2} \\
& \leqslant \lambda c_{f} w_{1}|u|^{q-2}-(r-1) h|u|^{r-2} \\
& \leqslant C \lambda w_{1}\left(\frac{\lambda w_{1}}{h}\right)^{(q-2) /(r-q)} .
\end{aligned}
$$

It follows that

$$
\begin{aligned}
\int_{\Omega}\left(\Xi\left(x, u_{n}\right)-\Xi\left(x, u_{0}\right)\right) \mathrm{d} x & =\int_{\Omega}\left(\int_{0}^{1} \int_{0}^{s} \xi_{u}\left(x, u_{0}+t\left(u_{n}-u_{0}\right)\right) \mathrm{d} t \mathrm{~d} s\right)\left(u_{n}-u_{0}\right)^{2} \mathrm{~d} x \\
& \leqslant C^{\prime} \int_{\Omega} \frac{w_{1}^{(r-2) /(r-q)}}{h^{(q-2) /(r-q)}}\left(u_{n}-u_{0}\right)^{2} \mathrm{~d} x .
\end{aligned}
$$

This inequality will be used to get the estimate for $\Phi_{\lambda, \theta}\left(u_{0}\right)-\Phi_{\lambda, \theta}\left(u_{n}\right)$ :

$$
\begin{aligned}
\Phi_{\lambda, \theta}\left(u_{0}\right)-\Phi_{\lambda, \theta}\left(u_{n}\right)= & \frac{1}{p}\left(\left\|u_{0}\right\|_{b}^{p}-\left\|u_{n}\right\|_{b}^{p}\right)+\theta \int_{\Gamma}\left(G\left(x, u_{n}\right)-G\left(x, u_{0}\right)\right) \mathrm{d} \Gamma \\
& +\int_{\Omega}\left(\Xi\left(x, u_{n}\right)-\Xi\left(x, u_{0}\right)\right) \mathrm{d} x \\
\leqslant & \frac{1}{p}\left(\left\|u_{0}\right\|_{b}^{p}-\left\|u_{n}\right\|_{b}^{p}\right)+\theta \int_{\Gamma}\left(G\left(x, u_{n}\right)-G\left(x, u_{0}\right)\right) \mathrm{d} \Gamma \\
& +C^{\prime} \int_{\Omega} \frac{w_{1}^{(r-2) /(r-q)}}{h^{(q-2) /(r-q)}}\left(u_{n}-u_{0}\right)^{2} \mathrm{~d} x .
\end{aligned}
$$

The compactness of the trace operator $E \rightarrow L^{m}\left(\Gamma ; w_{2}\right)$ and the continuity of the Nemytskii operator $N_{G}: L^{m}\left(\Gamma ; w_{2}\right) \rightarrow L^{1}(\Gamma)$ imply that $N_{G}\left(u_{n}\right) \rightarrow N_{G}\left(u_{0}\right)$ in $L^{1}(\Gamma)$, i.e. $\int_{\Gamma}\left|N_{G}\left(u_{n}\right)-N_{G}\left(u_{0}\right)\right| \mathrm{d} \Gamma \rightarrow 0$ as $n \rightarrow \infty$. It follows that

$$
\lim _{n \rightarrow \infty} \int_{\Gamma} G\left(x, u_{n}\right) \mathrm{d} \Gamma=\int_{\Gamma} G\left(x, u_{0}\right) \mathrm{d} \Gamma
$$


By Hölder's inequality we find

$\int_{\Omega} \frac{w_{1}^{(r-2) /(r-q)}}{h^{(q-2) /(r-q)}}\left(u_{n}-u_{0}\right)^{2} \mathrm{~d} x \leqslant\left(\int_{\Omega}\left(\frac{w_{1}^{r}}{h^{q}}\right)^{1 /(r-q)} \mathrm{d} x\right)^{(q-2) / q} \cdot\left(\int_{\Omega}\left|u_{n}-u_{0}\right|^{q} w_{1}(x) \mathrm{d} x\right)^{2 / q}$.

Since $u_{n} \rightarrow u_{0}$ in $L^{q}\left(\Omega ; w_{1}\right)$ we obtain

$$
\lim _{n \rightarrow \infty} \int_{\Omega} \frac{w_{1}^{(r-2) /(r-q)}}{h^{(q-2) /(r-q)}}\left(u_{n}-u_{0}\right)^{2} \mathrm{~d} x=0 .
$$

The lower semicontinuity of $\|\cdot\|_{b}$ with respect to the weak topology, (5.4) and (5.5) finish the proof.

Lemma 5.3. Suppose $\theta_{*}<\theta<\theta^{*}$ and $\lambda_{n} \searrow \lambda_{0}>0$ such that problem $\left(\mathrm{P}_{\lambda_{n}, \theta}\right)$ has a solution $u_{n}$ for each $n$. Then $\left\{u_{n}\right\}$ converges weakly (up to a subsequence) in $X$ to some $u_{0}$ which is a non-negative critical point of $\Phi_{\lambda_{0}, \theta}$.

Proof. By Corollary 4.1, $\left\{u_{n}\right\}$ is bounded in $X$. Therefore, in view of Remark 1.2, Lemma 3.2 and (2.1), we may assume (passing eventually to subsequences) that

$$
\begin{aligned}
& u_{n} \rightarrow u_{0} \text { in } X, \quad u_{n} \rightarrow u_{0} \text { in } L^{r}(\Omega ; h), \quad u_{n} \rightarrow u_{0} \text { in } E, \quad u_{n} \rightarrow u_{0} \text { in } L^{p}(\Gamma ; b), \\
& \frac{\partial u_{n}}{\partial x_{i}} \rightarrow \frac{\partial u_{0}}{\partial x_{i}} \text { in } L^{p}(\Omega ; a), \\
& u_{n} \rightarrow u_{0} \text { in } L^{q}\left(\Omega ; w_{1}\right), \quad u_{n} \rightarrow u_{0} \text { in } L^{m}\left(\Gamma ; w_{2}\right), \\
& u_{n} \rightarrow u_{0} \text { a.e. in } \Omega, \quad u_{n} \rightarrow u_{0} \text { a.e. in } \Gamma \text {. }
\end{aligned}
$$

We now observe that the embedding $E \hookrightarrow L_{\text {loc }}^{s}(\Omega)$ is compact for all $p \leqslant s<p^{*}$. This and (5.6) imply

$$
u_{n} \rightarrow u_{0} \text { in } L_{\text {loc }}^{s}(\Omega), \quad \forall p \leqslant s<p^{*} .
$$

Since $u_{n}$ is a non-negative critical point of $\Phi_{\lambda_{n}, \theta}$ for each $n$, we derive by (5.7) that $u_{0} \geqslant 0$ in $\Omega$ and for any $v \in X$ we have

$$
\begin{aligned}
\int_{\Omega} a\left|D u_{n}\right|^{p-2} D u_{n} \cdot D v \mathrm{~d} x+\int_{\Gamma} b\left|u_{n}\right|^{p-2} u_{n} v \mathrm{~d} \Gamma+\int_{\Omega} h\left|u_{n}\right|^{r-2} u_{n} v \mathrm{~d} x \\
=\int_{\Omega} f\left(\lambda_{n}, x, u_{n}\right) v \mathrm{~d} x+\theta \int_{\Gamma} g\left(x, u_{n}\right) v \mathrm{~d} \Gamma .
\end{aligned}
$$

By (5.6) we find that $\left\{\left|u_{n}\right|^{r-2} u_{n}\right\}$ is bounded in $L^{r /(r-1)}(\Omega ; h)$, while by (5.7) we have that $\left|u_{n}\right|^{r-2} u_{n} \rightarrow\left|u_{0}\right|^{r-2} u_{0}$ a.e. in $\Omega$. Combining these facts we get

$$
\left|u_{n}\right|^{r-2} u_{n} \rightarrow\left|u_{0}\right|^{r-2} u_{0} \text { in } L^{r /(r-1)}(\Omega ; h) .
$$

For $v \in L^{r}(\Omega ; h)$ fixed, set $l_{v}(u)=\int_{\Omega} h u v \mathrm{~d} x$, for all $u \in L^{r /(r-1)}(\Omega ; h)$. It is easy to verify that $l_{v} \in\left(L^{r /(r-1)}(\Omega ; h)\right)^{\prime}$. This together with (5.9) implies

$$
\lim _{n \rightarrow \infty} \int_{\Omega} h\left|u_{n}\right|^{r-2} u_{n} v \mathrm{~d} x=\int_{\Omega} h\left|u_{0}\right|^{r-2} u_{0} v \mathrm{~d} x, \quad \forall v \in X .
$$


Similarly,

$$
\lim _{n \rightarrow \infty} \int_{\Gamma} b\left|u_{n}\right|^{p-2} u_{n} v \mathrm{~d} \Gamma=\int_{\Gamma} b\left|u_{0}\right|^{p-2} u_{0} v \mathrm{~d} \Gamma, \quad \forall v \in X
$$

Taking into account (5.7) and Lemma 3.3 we infer that

$$
N_{f_{\lambda_{0}}}\left(u_{n}\right) \rightarrow N_{f_{\lambda_{0}}}\left(u_{0}\right) \text { in } L^{q /(q-1)}\left(\Omega ; w_{1}^{1 /(1-q)}\right)
$$

and

$$
N_{g}\left(u_{n}\right) \rightarrow N_{g}\left(u_{0}\right) \text { in } L^{m /(m-1)}\left(\Gamma ; w_{2}^{1 /(1-m)}\right) .
$$

By Hölder's inequality and $\left(\mathbf{H}_{\mathbf{7}}\right)$ we derive the estimates

$$
\begin{aligned}
& \int_{\Omega}\left|\left(f\left(\lambda_{n}, x, u_{n}\right)-f\left(\lambda_{0}, x, u_{0}\right)\right) v\right| \mathrm{d} x \\
& \leqslant \int_{\Omega}\left|\left(f\left(\lambda_{n}, x, u_{n}\right)-f\left(\lambda_{0}, x, u_{n}\right)\right) v\right| \mathrm{d} x+\int_{\Omega}\left|\left(f\left(\lambda_{0}, x, u_{n}\right)-f\left(\lambda_{0}, x, u_{0}\right)\right) v\right| \mathrm{d} x \\
& \leqslant C_{f}\left|\lambda_{n}-\lambda_{0}\right| \int_{\Omega}\left|u_{n}\right|^{q-1}|v| w_{1} \mathrm{~d} x+\int_{\Omega}\left|\left(N_{f_{\lambda_{0}}}\left(u_{n}\right)-N_{f_{\lambda_{0}}}\left(u_{0}\right)\right) v\right| \mathrm{d} x \\
& \leqslant C_{f}\left|\lambda_{n}-\lambda_{0}\right|\left\|u_{n}\right\|_{q, \Omega, w_{1}}^{q-1}\|v\|_{q, \Omega, w_{1}} \\
& \quad+\left\|N_{f_{\lambda_{0}}}\left(u_{n}\right)-N_{f_{\lambda_{0}}}\left(u_{0}\right)\right\|_{q /(q-1), \Omega, w_{1}^{1 /(1-q)}}\|v\|_{q, \Omega, w_{1}}
\end{aligned}
$$

and

$$
\int_{\Gamma}\left|\left(g\left(x, u_{n}\right)-g\left(x, u_{0}\right)\right) v\right| \mathrm{d} \Gamma \leqslant\left\|N_{g}\left(u_{n}\right)-N_{g}\left(u_{0}\right)\right\|_{m /(m-1), \Gamma, w_{2}^{1 /(1-m)}}\|v\|_{m, \Gamma, w_{2}} .
$$

Then, in virtue of (5.12) we find

$$
\left.\begin{array}{rlrl}
\lim _{n \rightarrow \infty} \int_{\Omega} f\left(\lambda_{n}, x, u_{n}\right) v \mathrm{~d} x & =\int_{\Omega} f\left(\lambda_{0}, x, u_{0}\right) v \mathrm{~d} x, & & \forall v \in X . \\
\lim _{n \rightarrow \infty} \int_{\Gamma} g\left(x, u_{n}\right) v \mathrm{~d} \Gamma=\int_{\Gamma} g\left(x, u_{0}\right) v \mathrm{~d} \Gamma, & & \forall v \in X .
\end{array}\right\}
$$

We now claim that $D u_{n} \rightarrow D u_{0}$ a.e. in $\Omega$. Set

$$
\Omega_{R}=\left\{x \in \mathbb{R}^{N}:|x|<R \text { and } \operatorname{dist}\left(x, \mathbb{R}^{N} \backslash \Omega\right)>\frac{1}{R}\right\} .
$$

It is clear that there exists $R_{0}>0$ such that $\Omega_{R} \neq \emptyset$ for all $R>R_{0}$. Since $\Omega_{R} \subset \Omega_{R^{\prime}} \subset \subset$ $\Omega$ for all $R_{0} \leqslant R<R^{\prime}$ and $\cup_{R \geqslant R_{0}} \Omega_{R}=\Omega$ we need only to show

$$
D u_{n} \rightarrow D u_{0} \quad \text { a.e. in } \Omega_{R} \text { for any } R \geqslant R_{0} .
$$


For this purpose we use the following inequalities (see [8, Lemma 4.10]) that hold for any $\xi, \zeta \in \mathbb{R}^{N}$

$$
\begin{array}{ll}
|\xi-\zeta|^{p} \leqslant C\left(|\xi|^{p-2} \xi-|\zeta|^{p-2} \zeta\right)(\xi-\zeta), & \text { for } p \geqslant 2 ; \\
|\xi-\zeta|^{2} \leqslant C\left(|\xi|^{p-2} \xi-|\zeta|^{p-2} \zeta\right)(\xi-\zeta)(|\xi|+|\zeta|)^{2-p}, & \text { for } 1<p<2 .
\end{array}
$$

Therefore, it is sufficient to prove that

$$
\left(\left|D u_{n}\right|^{p-2} D u_{n}-\left|D u_{0}\right|^{p-2} D u_{0}\right) \cdot\left(D u_{n}-D u_{0}\right) \rightarrow 0 \quad \text { a.e. in } \Omega_{R} \text { for any } R \geqslant R_{0} .
$$

For a fixed $R \geqslant R_{0}$, choose $\vartheta \in C_{0}^{\infty}\left(\mathbb{R}^{N}\right)$ with $0 \leqslant \vartheta \leqslant 1$ in $\mathbb{R}^{N}, \vartheta \equiv 1$ on $\Omega_{R}$ and $\vartheta \equiv 0$ on $\mathbb{R}^{N} \backslash \Omega_{2 R}$. Then by (5.6) and (5.7) we have that $\vartheta u_{n} \rightarrow \vartheta u_{0}$ in $E$ which yields

$$
\int_{\Omega} a\left|D u_{0}\right|^{p-2} D u_{0} \cdot D\left(\vartheta u_{n}-\vartheta u_{0}\right) \mathrm{d} x+\int_{\Gamma} b \vartheta\left|u_{0}\right|^{p-2} u_{0}\left(u_{n}-u_{0}\right) \mathrm{d} \Gamma \rightarrow 0 .
$$

By Hölder's inequality and (5.8) we find

$$
\begin{aligned}
& \left.\left|\int_{\Omega} a\left(u_{n}-u_{0}\right)\right| D u_{0}\right|^{p-2} D u_{0} \cdot D \vartheta \mathrm{d} x \mid \\
& \leqslant C_{1}\left(\int_{\operatorname{Supp} \vartheta} a\left|D u_{0}\right|^{p} \mathrm{~d} x\right)^{(p-1) / p}\left(\int_{\operatorname{Supp} \vartheta}\left|u_{n}-u_{0}\right|^{p} \mathrm{~d} x\right)^{1 / p} \rightarrow 0 .
\end{aligned}
$$

Using this fact in (5.18) we obtain

$$
\int_{\Omega} a \vartheta\left|D u_{0}\right|^{p-2} D u_{0} \cdot D\left(u_{n}-u_{0}\right) \mathrm{d} x+\int_{\Gamma} b \vartheta\left|u_{0}\right|^{p-2} u_{0}\left(u_{n}-u_{0}\right) \mathrm{d} \Gamma \rightarrow 0 .
$$

On the other hand, since $\left\langle\Phi_{\lambda_{n}, \theta}^{\prime}\left(u_{n}\right), \vartheta\left(u_{n}-u_{0}\right)\right\rangle=0$ we have

$$
\begin{gathered}
\int_{\Omega} a \vartheta\left|D u_{n}\right|^{p-2} D u_{n} \cdot D\left(u_{n}-u_{0}\right) \mathrm{d} x+\int_{\Gamma} b \vartheta\left|u_{n}\right|^{p-2} u_{n}\left(u_{n}-u_{0}\right) \mathrm{d} \Gamma \\
+\int_{\Omega} a\left(u_{n}-u_{0}\right)\left|D u_{n}\right|^{p-2} D u_{n} \cdot D \vartheta \mathrm{d} x=\int_{\Omega} h \vartheta\left|u_{n}\right|^{r-2} u_{n}\left(u_{0}-u_{n}\right) \mathrm{d} x \\
\quad+\int_{\Omega} f\left(\lambda_{n}, x, u_{n}\right) \vartheta\left(u_{n}-u_{0}\right) \mathrm{d} x+\theta \int_{\Gamma} g\left(x, u_{n}\right) \vartheta\left(u_{n}-u_{0}\right) \mathrm{d} \Gamma .
\end{gathered}
$$

By Hölder's inequality, (5.6) and (5.8) we derive

$$
\begin{aligned}
& \left.\left|\int_{\Omega} a\left(u_{n}-u_{0}\right)\right| D u_{n}\right|^{p-2} D u_{n} \cdot D \vartheta \mathrm{d} x \mid \\
& \leqslant C_{1}\left(\int_{\operatorname{Supp} \vartheta} a\left|D u_{n}\right|^{p} \mathrm{~d} x\right)^{(p-1) / p}\left(\int_{\operatorname{Supp} \vartheta}\left|u_{n}-u_{0}\right|^{p} \mathrm{~d} x\right)^{1 / p} \rightarrow 0
\end{aligned}
$$

and

$$
\begin{aligned}
& \left.\left|\int_{\Omega} h \vartheta\right| u_{n}\right|^{r-2} u_{n}\left(u_{0}-u_{n}\right) \mathrm{d} x \mid \\
& \leqslant C_{2}\left(\int_{\operatorname{Supp} \vartheta} h\left|u_{n}\right|^{r} \mathrm{~d} x\right)^{(r-1) / r}\left(\int_{\operatorname{Supp} \vartheta}\left|u_{n}-u_{0}\right|^{r} \mathrm{~d} x\right)^{1 / r} \rightarrow 0 .
\end{aligned}
$$


By (3.4), (5.7), (5.12) and Hölder's inequality we see that

$$
\begin{aligned}
\left|\int_{\Omega} f\left(\lambda_{n}, x, u_{n}\right) \vartheta\left(u_{n}-u_{0}\right) \mathrm{d} x\right| & \leqslant \tilde{C}_{f} \sup _{n \geqslant 1} \lambda_{n} \int_{\Omega}\left|u_{n}\right|^{q-1}\left|u_{n}-u_{0}\right| w_{1} \mathrm{~d} x \\
& \leqslant \tilde{C}_{f} \sup _{n \geqslant 1} \lambda_{n}\left\|u_{n}\right\|_{q, \Omega, w_{1}}^{q-1}\left\|u_{n}-u_{0}\right\|_{q, \Omega, w_{1}} \rightarrow 0
\end{aligned}
$$

and

$$
\left|\int_{\Gamma} g\left(x, u_{n}\right) \vartheta\left(u_{n}-u_{0}\right) \mathrm{d} \Gamma\right| \leqslant\left\|N_{g}\left(u_{n}\right)\right\|_{m /(m-1), \Gamma, w_{2}^{1 /(1-m)}}\left\|u_{n}-u_{0}\right\|_{m, \Gamma, w_{2}} \rightarrow 0 .
$$

It follows that

$$
\int_{\Omega} a \vartheta\left|D u_{n}\right|^{p-2} D u_{n} \cdot D\left(u_{n}-u_{0}\right) \mathrm{d} x+\int_{\Gamma} b \vartheta\left|u_{n}\right|^{p-2} u_{n}\left(u_{n}-u_{0}\right) \mathrm{d} \Gamma \rightarrow 0
$$

Since

$$
\begin{gathered}
0 \leqslant \int_{\Omega} a \vartheta\left(\left|D u_{n}\right|^{p-2} D u_{n}-\left|D u_{0}\right|^{p-2} D u_{0}\right) \cdot\left(D u_{n}-D u_{0}\right) \mathrm{d} x \\
\leqslant \int_{\Omega} a \vartheta\left(\left|D u_{n}\right|^{p-2} D u_{n}-\left|D u_{0}\right|^{p-2} D u_{0}\right) \cdot\left(D u_{n}-D u_{0}\right) \mathrm{d} x \\
\quad+\int_{\Gamma} b \vartheta\left(\left|u_{n}\right|^{p-2} u_{n}-\left|u_{0}\right|^{p-2} u_{0}\right)\left(u_{n}-u_{0}\right) \mathrm{d} \Gamma
\end{gathered}
$$

we deduce by (5.19) and (5.20) that

$$
\lim _{n \rightarrow \infty} \int_{\Omega_{R}} a\left(\left|D u_{n}\right|^{p-2} D u_{n}-\left|D u_{0}\right|^{p-2} D u_{0}\right) \cdot\left(D u_{n}-D u_{0}\right) \mathrm{d} x=0 .
$$

Hence (5.17) holds. Therefore, the claim that $D u_{n} \rightarrow D u_{0}$ a.e. in $\Omega$ is proved. This combined with the fact that $\left\{\left|D u_{n}\right|^{p-2} \partial u_{n} / \partial x_{i}\right\}$ is bounded in $L^{p /(p-1)}(\Omega ; a)$ implies

$$
\left|D u_{n}\right|^{p-2} \frac{\partial u_{n}}{\partial x_{i}} \rightarrow\left|D u_{0}\right|^{p-2} \frac{\partial u_{0}}{\partial x_{i}} \quad \text { in } L^{p /(p-1)}(\Omega ; a) .
$$

It follows that

$$
\lim _{n \rightarrow \infty} \int_{\Omega} a\left|D u_{n}\right|^{p-2} D u_{n} \cdot D v \mathrm{~d} x=\int_{\Omega} a\left|D u_{0}\right|^{p-2} D u_{0} \cdot D v \mathrm{~d} x, \quad \forall v \in X .
$$

By (5.10), (5.11), (5.14) and (5.21) we conclude that $u_{0}$ is a critical point of $\Phi_{\lambda_{0}, \theta}$.

\section{Proof of Theorem 2.3}

Let $\theta \in J$ and $\lambda>0$ be arbitrary. From Lemma 5.1 we see that $m_{\lambda, \theta}:=\inf _{u \in X} \Phi_{\lambda, \theta}(u)$ is real. Let $\left\{u_{n}\right\}$ be a sequence such that $\lim _{n \rightarrow \infty} \Phi_{\lambda, \theta}\left(u_{n}\right)=m_{\lambda, \theta}$. According to Lemma 5.2, we can assume (up to a subsequence) that

$$
u_{n} \rightarrow u_{0} \text { in } X \quad \text { and } \quad \Phi_{\lambda, \theta}\left(u_{0}\right) \leqslant \liminf _{n \rightarrow \infty} \Phi_{\lambda, \theta}\left(u_{n}\right)=m_{\lambda, \theta} .
$$


This shows that $\inf _{u \in X} \Phi_{\lambda, \theta}(u)$ is attained in $u_{0}$. From $\left(\mathbf{H}_{\mathbf{4}}\right)$ and $\left(\mathbf{H}_{\mathbf{8}}\right)$ we deduce that $G\left(x,\left|u_{0}\right|\right) \geqslant G\left(x, u_{0}\right)$ a.e. $x \in \Gamma$ and $F\left(\lambda, x,\left|u_{0}\right|\right) \geqslant F\left(\lambda, x, u_{0}\right)$ a.e. $x \in \Omega$. It follows that $\Phi_{\lambda, \theta}\left(\left|u_{0}\right|\right) \leqslant \Phi_{\lambda, \theta}\left(u_{0}\right)$. Therefore, we may assume that $u_{0} \geqslant 0$ on $\Omega$. To ensure that $u_{0} \not \equiv 0$ we shall prove that $m_{\lambda, \theta}$ is negative provided that $\lambda>\tilde{\lambda}$ for some $\tilde{\lambda}>0$.

By hypothesis $\left(\mathbf{H}_{\mathbf{6}}\right)$ we deduce that there exists $\delta>0$ independent of $x$ and $\lambda$ such that

$$
F(\lambda, x, u(x)) \geqslant \frac{\lambda}{2 q}|u(x)|^{q} w_{1}(x) \quad \text { a.e. } x \in \Omega, \forall u \in X \text { with } \sup _{x \in \Omega}|u(x)| \leqslant \delta .
$$

Set $\zeta>0$ with the property that

$$
Y=\left\{u \in X \backslash\{0\}: \sup _{x \in \Omega}|u(x)| \leqslant \zeta\|u\|_{q, \Omega, w_{1}}\right\} \neq \emptyset
$$

and denote $\eta=(\delta / \zeta)^{q}$. Define

$$
\tilde{\lambda}:=\inf \left\{\frac{2 q}{\eta p}\|u\|_{b}^{p}-\frac{2 q}{\eta} \theta \int_{\Gamma} G(x, u) \mathrm{d} \Gamma+\frac{2 q}{\eta r} \int_{\Omega} h|u|^{r} \mathrm{~d} x: u \in Z\right\},
$$

where

$$
Z=\left\{u \in X: \sup _{x \in \Omega}|u(x)| \leqslant \delta, \int_{\Omega}|u|^{q} w_{1}(x) \mathrm{d} x=\eta\right\} .
$$

It is easy to verify that $Z \neq \emptyset$. Indeed, if $y \in Y$, then

$$
u=\frac{\eta^{1 / q}}{\|y\|_{q, \Omega, w_{1}}} y \in Z
$$

We now claim that $\tilde{\lambda}>0$. For this aim, we consider the constrained minimization problem,

$$
M:=\inf \left\{\|u\|_{b}^{p}: u \in E, \int_{\Omega}|u|^{q} w_{1}(x) \mathrm{d} x=\eta\right\} .
$$

Since the embedding $E \hookrightarrow L^{q}\left(\Omega ; w_{1}\right)$ is continuous, it follows that $M>0$. Thus

$$
\|u\|_{b}^{p} \geqslant M \quad \text { for all } u \in X \text { with } \int_{\Omega}|u|^{q} w_{1}(x) \mathrm{d} x=\eta .
$$

By applying the Hölder inequality we find

$$
\int_{\Omega}|u|^{q} w_{1} \mathrm{~d} x=\int_{\Omega} \frac{w_{1}}{h^{q / r}} h^{q / r}|u|^{q} \mathrm{~d} x \leqslant\left(\int_{\Omega}\left(\frac{w_{1}^{r}}{h^{q}}\right)^{1 /(r-q)} \mathrm{d} x\right)^{(r-q) / r} \cdot\left(\int_{\Omega} h|u|^{r} \mathrm{~d} x\right)^{q / r} .
$$

By virtue of (5.1) and (6.2) we have

$$
\begin{aligned}
\frac{2 q}{\eta p}\|u\|_{b}^{p}-\frac{2 q}{\eta} \theta \int_{\Gamma} G(x, u) & \mathrm{d} \Gamma+\frac{2 q}{\eta r} \int_{\Omega} h|u|^{r} \mathrm{~d} x \\
& \geqslant \frac{2 q}{\eta} c\|u\|_{b}^{p}+\frac{2 q}{\eta r} \int_{\Omega} h|u|^{r} \mathrm{~d} x \\
& \geqslant \frac{2 q}{\eta} c M+\frac{2 q}{\eta r} \eta^{r / q}\left(\int_{\Omega}\left(\frac{w_{1}^{r}}{h^{q}}\right)^{1 /(r-q)} \mathrm{d} x\right)^{-(r-q) / q}
\end{aligned}
$$


for all $u \in X$ with $\int_{\Omega}|u|^{q} w_{1} \mathrm{~d} x=\eta$. It follows that

$$
\tilde{\lambda} \geqslant \frac{2 q}{\eta} c M+\frac{2 q}{r} \eta^{(r-q) / q}\left(\int_{\Omega}\left(\frac{w_{1}^{r}}{h^{q}}\right)^{1 /(r-q)} \mathrm{d} x\right)^{-(r-q) / q}
$$

and our claim follows.

Let $\lambda>\tilde{\lambda}$. Then there exists a function $u_{1} \in Z$ such that

$$
\lambda>\frac{2 q}{\eta p}\left\|u_{1}\right\|_{b}^{p}-\frac{2 q}{\eta} \theta \int_{\Gamma} G\left(x, u_{1}\right) \mathrm{d} \Gamma+\frac{2 q}{\eta r} \int_{\Omega} h\left|u_{1}\right|^{r} \mathrm{~d} x .
$$

This inequality and (6.1) imply

$$
\begin{aligned}
\Phi_{\lambda, \theta}\left(u_{1}\right) & =\frac{1}{p}\left\|u_{1}\right\|_{b}^{p}-\theta \int_{\Gamma} G\left(x, u_{1}\right) \mathrm{d} \Gamma+\frac{1}{r} \int_{\Omega} h\left|u_{1}\right|^{r} \mathrm{~d} x-\int_{\Omega} F\left(\lambda, x, u_{1}(x)\right) \mathrm{d} x \\
& \leqslant \frac{1}{p}\left\|u_{1}\right\|_{b}^{p}-\theta \int_{\Gamma} G\left(x, u_{1}\right) \mathrm{d} \Gamma+\frac{1}{r} \int_{\Omega} h\left|u_{1}\right|^{r} \mathrm{~d} x-\frac{\lambda}{2 q} \int_{\Omega}\left|u_{1}\right|^{q} w_{1} \mathrm{~d} x<0 .
\end{aligned}
$$

Consequently, $\inf _{u \in X} \Phi_{\lambda, \theta}(u)<0$. Thus, the problem $\left(\mathrm{P}_{\lambda, \theta}\right)$ has a solution if $\theta \in J$ and $\lambda>\tilde{\lambda}$. Set

$$
\lambda_{0}=\inf \left\{\lambda>0:\left(\mathrm{P}_{\lambda, \theta}\right) \text { admits a solution }\right\} .
$$

By Theorem 2.2, we see that $\lambda_{0} \geqslant \lambda^{*}>0$.

We now show that for each $\lambda>\lambda_{0}$ problem $\left(\mathrm{P}_{\lambda, \theta}\right)$ admits a solution. Indeed, for every $\lambda>\lambda_{0}$ there exists $\rho \in\left(\lambda_{0}, \lambda\right)$ such that problem $\left(\mathrm{P}_{\rho, \theta}\right)$ has a solution $u_{\rho}$ which is a subsolution of problem $\left(\mathrm{P}_{\lambda, \theta}\right)$. We consider the variational problem,

$$
\inf \left\{\Phi_{\lambda, \theta}(u): u \in X \text { and } u \geqslant u_{\rho}\right\} .
$$

By Lemmas 5.1 and 5.2 this problem admits a solution $\bar{u}$. This minimizer $\bar{u}$ is a solution of problem $\left(\mathrm{P}_{\lambda, \theta}\right)$. It remains to show that problem $\left(\mathrm{P}_{\lambda_{0}, \theta}\right)$ also has a solution. Let $\lambda_{n} \rightarrow \lambda_{0}$ and $\lambda_{n}>\lambda_{0}$ for each $n$. Problem $\left(\mathrm{P}_{\lambda_{n}, \theta}\right)$ has a solution $u_{n}$ for each $n$. Then, in virtue of Lemma 5.3, we may assume (up to a subsequence) that $u_{n} \rightarrow u_{0}$ in $X$, $u_{n} \rightarrow u_{0}$ in $L^{q}\left(\Omega ; w_{1}\right), u_{n} \rightarrow u_{0}$ in $L^{m}\left(\Gamma ; w_{2}\right)$, where $u_{0}$ is a non-negative critical point of $\Phi_{\lambda_{0}, \theta}$. To conclude that $u_{0}$ is a solution of problem $\left(\mathrm{P}_{\lambda_{0}, \theta}\right)$ it remains only to prove that $u_{0} \not \equiv 0$. Since $u_{n}$ and $u_{0}$ are critical points of $\left(\Phi_{\lambda_{n}, \theta}\right)$ and $\left(\Phi_{\lambda_{0}, \theta}\right)$, respectively, we have

$$
\left\langle I^{\prime}\left(u_{n}\right), u_{n}-u_{0}\right\rangle-\left\langle I^{\prime}\left(u_{0}\right), u_{n}-u_{0}\right\rangle+\left\langle J^{\prime}\left(u_{n}\right), u_{n}-u_{0}\right\rangle-\left\langle J^{\prime}\left(u_{0}\right), u_{n}-u_{0}\right\rangle=J_{1, n}+J_{2, n},
$$

where

$$
\begin{aligned}
& J_{1, n}=\int_{\Omega}\left(f\left(\lambda_{n}, x, u_{n}\right)-f\left(\lambda_{0}, x, u_{0}\right)\right)\left(u_{n}-u_{0}\right) \mathrm{d} x, \\
& J_{2, n}=\theta \int_{\Gamma}\left(g\left(x, u_{n}\right)-g\left(x, u_{0}\right)\right)\left(u_{n}-u_{0}\right) \mathrm{d} \Gamma .
\end{aligned}
$$


It is easy to see that

$$
0 \leqslant\left\langle I^{\prime}\left(u_{n}\right), u_{n}-u_{0}\right\rangle-\left\langle I^{\prime}\left(u_{0}\right), u_{n}-u_{0}\right\rangle \leqslant J_{1, n}+J_{2, n} .
$$

Using (3.4) we get the estimate

$$
\left|J_{1, n}\right| \leqslant \tilde{C}_{f}\left(\lambda_{n} \int_{\Omega}\left|u_{n}\right|^{q-1}\left|u_{n}-u_{0}\right| w_{1}(x) \mathrm{d} x+\lambda_{0} \int_{\Omega}\left|u_{0}\right|^{q-1}\left|u_{n}-u_{0}\right| w_{1}(x) \mathrm{d} x\right)
$$

and it follows from the Hölder inequality that

$$
\left|J_{1, n}\right| \leqslant \tilde{C}_{f}\left(\sup _{n \geqslant 1} \lambda_{n}\left\|u_{n}\right\|_{q, \Omega, w_{1}}^{q-1}+\lambda_{0}\left\|u_{0}\right\|_{q, \Omega, w_{1}}^{q-1}\right)\left\|u_{n}-u_{0}\right\|_{q, \Omega, w_{1}} \rightarrow 0 .
$$

By (5.12) and Hölder's inequality we find

$$
\left|J_{2, n}\right| \leqslant|\theta|\left\|N_{g}\left(u_{n}\right)-N_{g}\left(u_{0}\right)\right\|_{m /(m-1), \Gamma, w_{2}^{1 /(1-m)}}\left\|u_{n}-u_{0}\right\|_{m, \Gamma, w_{2}} \rightarrow 0 .
$$

Relations (6.3), (6.4) and (6.5) yield

$$
\left\langle I^{\prime}\left(u_{n}\right), u_{n}-u_{0}\right\rangle-\left\langle I^{\prime}\left(u_{0}\right), u_{n}-u_{0}\right\rangle \rightarrow 0 \quad \text { as } n \rightarrow \infty .
$$

We show that $\left\|u_{n}-u_{0}\right\|_{b} \rightarrow 0$ as $n \rightarrow \infty$. We distinguish two cases which may occur.

Case 1. $p \geqslant 2$. Using (5.15) we obtain

$$
\left\|u_{n}-u_{0}\right\|_{b}^{p} \leqslant C\left(\left\langle I^{\prime}\left(u_{n}\right), u_{n}-u_{0}\right\rangle-\left\langle I^{\prime}\left(u_{0}\right), u_{n}-u_{0}\right\rangle\right) \rightarrow 0 \quad \text { as } n \rightarrow \infty,
$$

which shows that $\left\|u_{n}\right\|_{b} \rightarrow\left\|u_{0}\right\|_{b}$ as $n \rightarrow \infty$.

Case 2. $1<p<2$. We observe that it is enough to show that

$$
\left\|u_{n}-u_{0}\right\|_{b}^{2} \leqslant C^{\prime}\left(\left\langle I^{\prime}\left(u_{n}\right), u_{n}-u_{0}\right\rangle-\left\langle I^{\prime}\left(u_{0}\right), u_{n}-u_{0}\right\rangle\right)\left(\left\|u_{n}\right\|_{b}^{2-p}+\left\|u_{0}\right\|_{b}^{2-p}\right) .
$$

In order to prove (6.6) we recall the following result: for all $s>0$ there is a constant $C_{s}>0$ such that

$$
(x+y)^{s} \leqslant C_{s}\left(x^{s}+y^{s}\right) \text { for any } x, y \in(0, \infty) .
$$

Then we obtain

$$
\begin{aligned}
\left\|u_{n}-u_{0}\right\|_{b}^{2} & =\left(\int_{\Omega} a(x)\left|D u_{n}-D u_{0}\right|^{p} \mathrm{~d} x+\int_{\Gamma} b(x)\left|u_{n}-u_{0}\right|^{p} \mathrm{~d} \Gamma\right)^{2 / p} \\
& \leqslant C_{p}\left[\left(\int_{\Omega} a(x)\left|D u_{n}-D u_{0}\right|^{p} \mathrm{~d} x\right)^{2 / p}+\left(\int_{\Gamma} b(x)\left|u_{n}-u_{0}\right|^{p} \mathrm{~d} \Gamma\right)^{2 / p}\right] .
\end{aligned}
$$


Using (5.16), (6.7) and the Hölder inequality we find

$$
\begin{aligned}
\int_{\Omega} a(x) \mid D u_{n}- & \left.D u_{0}\right|^{p} \mathrm{~d} x \\
= & \int_{\Omega} a(x)\left(\left|D u_{n}-D u_{0}\right|^{2}\right)^{p / 2} \mathrm{~d} x \\
\leqslant & c_{1} \int_{\Omega}\left(a\left(\left|D u_{n}\right|^{p-2} D u_{n}-\left|D u_{0}\right|^{p-2} D u_{0}\right) \cdot\left(D u_{n}-D u_{0}\right)\right)^{p / 2} \\
& \times\left(a\left(\left|D u_{n}\right|+\left|D u_{0}\right|\right)^{p}\right)^{(2-p) / 2} \mathrm{~d} x \\
\leqslant & c_{1}\left(\int_{\Omega} a\left(\left|D u_{n}\right|^{p-2} D u_{n}-\left|D u_{0}\right|^{p-2} D u_{0}\right) \cdot\left(D u_{n}-D u_{0}\right) \mathrm{d} x\right)^{p / 2} \\
& \times\left(\int_{\Omega} a\left(\left|D u_{n}\right|+\left|D u_{0}\right|\right)^{p} \mathrm{~d} x\right)^{(2-p) / 2} \\
\leqslant & c_{2}\left(\int_{\Omega} a\left(\left|D u_{n}\right|^{p-2} D u_{n}-\left|D u_{0}\right|^{p-2} D u_{0}\right)\left(D u_{n}-D u_{0}\right) \mathrm{d} x\right)^{p / 2} \\
& \times\left(\int_{\Omega}\left(a\left|D u_{n}\right|^{p}+a\left|D u_{0}\right|^{p}\right) \mathrm{d} x\right)^{(2-p) / 2} \\
\leqslant & c_{3}\left(\int_{\Omega} a(x)\left(\left|D u_{n}\right|^{p-2} D u_{n}-\left|D u_{0}\right|^{p-2} D u_{0}\right) \cdot\left(D u_{n}-D u_{0}\right) \mathrm{d} x\right)^{p / 2} \\
& \times\left(\left\|u_{n}\right\|_{b}^{p}+|| u_{0} \|_{b}^{p}\right)^{(2-p) / 2} \\
\leqslant & c_{4}\left(\int_{\Omega} a(x)\left(\left|D u_{n}\right|^{p-2} D u_{n}-\left|D u_{0}\right|^{p-2} D u_{0}\right) \cdot\left(D u_{n}-D u_{0}\right) \mathrm{d} x\right)^{p / 2} \\
& \times\left(\left\|u_{n}\right\|_{b}^{(2-p) p / 2}+\left\|u_{0}\right\|_{b}^{(2-p) p / 2}\right) .
\end{aligned}
$$

Using the last inequality and (6.7) we have the estimate,

$$
\begin{aligned}
& \left(\int_{\Omega} a(x)\left|D u_{n}-D u_{0}\right|^{p} \mathrm{~d} x\right)^{2 / p} \\
& \quad \leqslant c_{p}\left(\left\langle I^{\prime}\left(u_{n}\right), u_{n}-u_{0}\right\rangle-\left\langle I^{\prime}\left(u_{0}\right), u_{n}-u_{0}\right\rangle\right)\left(\left\|u_{n}\right\|_{b}^{2-p}+\left\|u_{0}\right\|_{b}^{2-p}\right) .
\end{aligned}
$$

In a similar way we can obtain the estimate,

$$
\begin{aligned}
& \left(\int_{\Gamma} b(x)\left|u_{n}-u_{0}\right|^{p} \mathrm{~d} \Gamma\right)^{2 / p} \\
& \quad \leqslant c_{p}^{\prime}\left(\left\langle I^{\prime}\left(u_{n}\right), u_{n}-u_{0}\right\rangle-\left\langle I^{\prime}\left(u_{0}\right), u_{n}-u_{0}\right\rangle\right)\left(\left\|u_{n}\right\|_{b}^{2-p}+\left\|u_{0}\right\|_{b}^{2-p}\right) .
\end{aligned}
$$

It is now easy to observe that inequalities (6.8), (6.9) and (6.10) imply the estimate (6.6).

In both cases, by Corollary $4.1, u_{0} \not \equiv 0$. This concludes our proof. 


\section{References}

1. S. Alama and G. Tarantello, Elliptic problems with nonlinearities indefinite in sign, J. Funct. Analysis 141 (1996), 159-215.

2. G. Aronsson and U. Janfalk, On Hele-Shaw flow of power-law fluids, Eur. J. Appl. Math. 3 (1992), 343-366.

3. T. Bhattacharya, E. DiBenedetto and J. Manfredi, Limits as $p \rightarrow \infty$ of $\Delta_{p} u_{p}=f$ and related extremal problems, Rend. Sem. Mat. Univ. Pol. Torino (Fascicolo Speciale), (1989), 15-68.

4. K. J. Brown and F. A. Davidson, Global bifurcation in the Brusselator system, Nonlinear Analysis 24 (1995), 1713-1725.

5. F. Cîrstea, D. Motreanu and V. RĂdulescu, Weak solutions of quasilinear problems with nonlinear boundary condition, Nonlinear Analysis 43 (2001), 623-636.

6. F. CîRSTEA AND V. RĂDulEsCU, Existence and nonexistence results for quasilinear problems with nonlinear boundary condition, J. Math. Analysis Appl. 244 (2000), 169-183.

7. R. Dautray And J.-L. Lions, Physical origins and classical methods, Mathematical Analysis and Numerical Methods for Science and Technology, vol. 1 (Springer, 1985).

8. J. I. DIAZ, Nonlinear partial differential equations and free boundaries. Elliptic equations, (Boston: Pitman, 1986).

9. P. DRÁBEK AND Y. X. HuAng, Positive solutions and bifurcations problems for some quasilinear elliptic equations in $\mathbb{R}^{N}$, Nonlinear Analysis 30 (1997), 3671-3681.

10. P. DRÁBEK AND C. Simader, Nonlinear eigenvalue problems for quasilinear equations on unbounded domains, Math. Nachr. 203 (1999), 5-30.

11. W. L. FindLEy, J. S. LAI AND K. ONARAN, Creep and relaxation of nonlinear viscoelastic materials (North Holland, 1976).

12. L. M. KaCHANOv, Foundations of the theory of plasticity (North Holland, 1971).

13. L. M. Kachanov, Crack growth under creep conditions, Mech. Solids 13 (1978), 89-94.

14. B. Kawohl, On a family of torsional creep problems, J. Reine Angew. Math. 410 (1990), $1-22$.

15. M. C. PÉlissier And M. L. Reynaud, Étude d'un modèle mathématique d'écoulement de glacier, C. R. Acad. Sci. Paris Sér. 1279 (1974), 531-534.

16. K. PFLÜGER, Existence and multiplicity of solutions to a $p$-Laplacian equation with nonlinear boundary condition, Electron. J. Diff. Eqns 10 (1998), 1-13.

17. K. PflüGER, Compact traces in weighted Sobolev spaces, Analysis 18 (1998), 65-83.

18. K. PflüGER, Nonlinear boundary value problems in weighted Sobolev spaces, Nonlinear Analysis 30 (1997), 1263-1270.

19. R. E. Showalter And N. J. WAlkington, Diffusion of fluid in a fissured medium with microstructure, SIAM J. Math. Analysis 22 (1991), 1702-1722.

20. A. Torchinsky, Real variable methods in harmonic analysis (Academic, 1986).

21. M. M. VAInBerG, Variational methods for the study of nonlinear operators (San Francisco: Holden Day, 1964). 